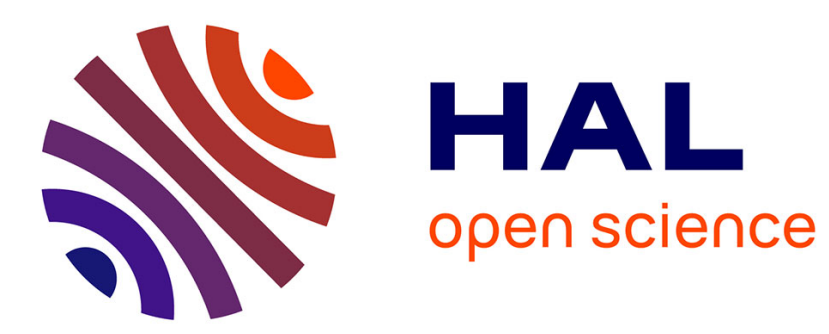

\title{
Non-modal linear stability analysis of ablation flows relative to inertial confinement fusion
}

G Varillon, J.-M Clarisse, A. Couairon

\section{To cite this version:}

G Varillon, J.-M Clarisse, A. Couairon. Non-modal linear stability analysis of ablation flows relative to inertial confinement fusion. IUTAM Transition 2019, International Union of Theoretical and Applied Mechanics, Sep 2019, Londres, United Kingdom. hal-02615043

\section{HAL Id: hal-02615043 \\ https://hal-polytechnique.archives-ouvertes.fr/hal-02615043}

Submitted on 22 May 2020

HAL is a multi-disciplinary open access archive for the deposit and dissemination of scientific research documents, whether they are published or not. The documents may come from teaching and research institutions in France or abroad, or from public or private research centers.
L'archive ouverte pluridisciplinaire HAL, est destinée au dépôt et à la diffusion de documents scientifiques de niveau recherche, publiés ou non, émanant des établissements d'enseignement et de recherche français ou étrangers, des laboratoires publics ou privés. 


\title{
Non-modal linear stability analysis of ablation flows relative to inertial confinement fusion
}

\author{
G. Varillon, J.-M. Clarisse and A. Couairon
}

\section{Introduction}

Inertial confinement fusion (ICF) aims at achieving controlled thermonuclear burn by means of a sufficiently symmetric implosion of a spherical pellet, under the action of an external radiation flux [1]. Such implosions are triggered and driven by the deflagration, or ablation, heat wave that thrusts inwardly the pellet outer shell, the ablator, chosen to be opaque to the incoming irradiation. The associated flow consists of a subsonic heat front, or ablation front, coinciding with the leading edge of the heated material expansion wave, that penetrates into the ablator, preceded by a fore-running shock wave [1, chap. 2], [2, vol. 2, chap. 10, §8]. Inherently unsteady, these ablation flows which undergo different regimes of acceleration during the implosion, are compressible and highly nonuniform with a steep heat front, owing to the strong nonlinearity of the heat transport and the intense incident heating.

Right from the beginning of research on the subject back in the 1970s, the hydrodynamic stability of ablation fronts has been identified as one of the most critical issues to the success of ICF. Since then, an impressive amount of efforts, in terms of modeling, numerical simulations, reduced- and full-scale laboratory experiments, has been devoted to the prediction, measurement and mitigation of hydrodynamic instabilities in ICF implosions and, in particular, in ICF ablation flows. Despite these efforts, thermonuclear ignition experiments carried out on the largest existing ICF

G. Varillon

CEA, DAM, DIF, F-91297 Arpajon, France,

CPHT, CNRS, École Polytechnique, Institut Polytechnique de Paris, F-91128 Palaiseau, France e-mail: gregoire.varillon@ polytechnique.edu

J.-M. Clarisse

CEA, DAM, DIF, F-91297 Arpajon, France e-mail: jean-marie.clarisse@ cea.fr

A. Couairon

CPHT, CNRS, École Polytechnique, Institut Polytechnique de Paris, F-91128 Palaiseau, France e-mail: arnaud.couairon@polytechnique.edu 
facility have not been yet conclusive [3]. Theoretical investigations of ablation flow stability have relied on simplifying assumptions that are not necessarily met in actual cases: e.g. mean-flow steadiness, low Mach-number approximation, unbounded domains, discontinuous ablation front, flow region uniformity of some sort [4]. In addition, these works have exclusively employed the method of normal modes, focusing on the least stable eigensolutions and thus only furnishing asymptotic stability results. More realistic configurations are classically handled by means of multidimensional numerical simulations carried out with ICF hydrodynamics codes which incorporate the most relevant physical phenomena. These "full-physics" simulations which are computationally very demanding, are necessarily of limited accuracy for decreasing perturbation scales and also suffer from overly dissipative and dispersive numerical schemes used in such hydrocodes. Such simulations consist in computing perturbation amplifications from selected perturbed initial or boundary conditions that are considered to be the "most dangerous" for a given mean-flow configuration. Comparisons with theoretical results and specifically designed experiments where a dominant most dangerous perturbation source is selected by a careful control of experimental conditions, have helped building confidence in the ability of ICF hydrocodes to reproduce instability dynamics [5, 6]. However, this approach faces a major difficulty in having to deal, in actual ICF implosions, with multiple perturbation sources whose contributions remain, for some of them, unsufficiently known and controlled. This fact is substantiated by recent successively targeted series of experiments which have revealed the detrimental effects of perturbation sources previously held to be minor, thus questioning the common acceptation of most dangerous perturbation sources for ICF pellet implosions.

In this context, methods of non-modal stability theory [7], being capable of identifying the most dangerous perturbations in time-dependent flows of finite durations, not only appear to be most valuable but also to be a necessity. Since the current cost of full-physics simulations forbids the implementation of such methods with existing ICF hydrocodes, we have recourse to a simplified modeling of unsteady ablation waves provided by self-similar solutions to the Euler equations with nonlinear heat conduction [8]. Some of these solutions are representative of the first stage of a pellet implosion, or shock transit phase, during which the fore-running shock wave is still traveling inside the ablator $[9,10]$. In particular these self-similar flows present the complete structure of an ablation wave: a leading shock front, a quasiisentropic compression (post-shock) region, an ablation layer and an expansion wave where heat conduction dominates (the conduction region): cf. Fig. 1. This modeling which does not give up essential features of ICF ablation waves (unsteadiness, compressibility, stratification and confinement) has previously been used to obtain linear perturbation responses for configurations of ICF commonly held as most dangerous $[11,12]$. Here we perform a non-modal stability analysis that consists in finding optimal initial perturbations (OIPs), i.e. initial perturbations that maximize a semi-norm of the flow perturbations at a given time horizon. Having to deal with time-dependent and non-uniform base flows, these OIPs are obtained by means of a direct-adjoint method that we have developed based on our previous experience with linear perturbation computations in such flows. 


\section{Model}

The chosen ablation wave modeling considers a semi-infinite slab of a polytropic gas subject to an incident radiation flux and a material pressure exerted at its external boundary. The gas is assumed to remain at local thermodynamic equilibrium and optically thick to the incoming radiation, allowing us to use a fluid model and the radiation heat-conduction approximation of radiative transfer to describe its motion. Under the conditions of ICF pellet irradiation, the external radiation flux is sufficiently high so that radiation heat conduction dominates any other diffusive effect within the fluid (e.g. viscosity) while the fluid temperature remains sufficiently low for radiation pressure and radiation energy to be negligible compared to material pressure and internal energy. Consequently, the equations of motion come down to the Euler equations with nonlinear heat conduction [2].

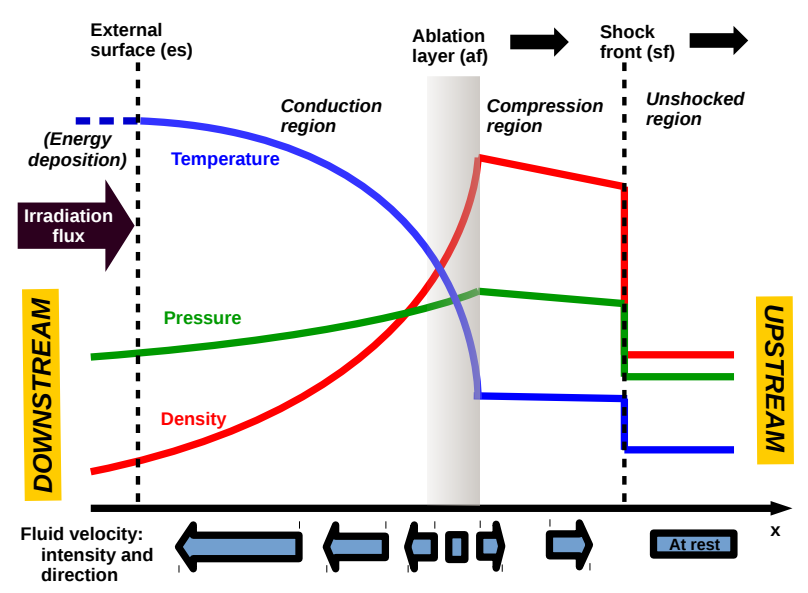

Fig. 1 Structure of an ablation wave.

\subsection{Base flow}

Assuming that the flow is along the $x$ direction of a Cartesian system of coordinates $(O, x, y, z)$, the equations of motion come, in dimensionless form and in the Lagrangian coordinate $m$ such that $\mathrm{d} m=\rho \mathrm{d} x$, as [9]

$$
\partial_{t} 1 / \rho-\partial_{m} v=0, \quad \partial_{t} v+\partial_{m} p=0, \quad \partial_{t}\left(e+v^{2} / 2\right)+\partial_{m}(p v+\varphi)=0
$$

where $\rho, v, p, e$ and $\varphi$ denote, respectively, the fluid density, velocity, pressure, specific internal energy, and radiation heat flux of expression

$$
\varphi=-\rho^{-\mu} T^{\nu} \rho \partial_{m} T \equiv \Psi\left(\rho, T, \partial_{x} T\right), \quad \text { with }-\mu=2, v=13 / 2,
$$


according to a radiation conduction model for a fully-ionized gas [2]. These equations are closed by the dimensionless equation of state

$$
p=\rho T, \quad e=C_{v} T, \quad C_{v}=1 /(\gamma-1),
$$

where $\gamma$ is the adiabatic gas exponent. Self-similar reduction of (1)-(3) arises if the incident heat flux and pressure at the external surface follow specific power laws $[8,13]$, namely

$$
\varphi(0, t)=\mathcal{B}_{\phi} t^{3 \alpha-3}, p(0, t)=\mathcal{B}_{p} t^{2 \alpha-2}, \text { for } t>0, \text { with } \alpha=\frac{2 v-1}{2 v-2},
$$

and for an initial state given by $(\rho, v, T)=(1,0,0)$ for $m \geq 0$. For certain values of the boundary parameters $\left(\mathcal{B}_{p}, \mathcal{B}_{\varphi}\right)$, these self-similar solutions reproduce the features of ICF ablation waves [9, 10]. Highly accurate solutions to (1)-(4) are obtained by means of an adaptive multidomain Chebyshev spectral method [14].

\subsection{Linear perturbations}

Three-dimensional linear perturbations of the above self-similar ablative waves are considered using an Eulerian description in the coordinate system $(m, y, z)$. The resulting system of partial differential equations (PDEs) in physical space is replaced by a system of one-dimensional PDEs in the $y z$-Fourier space. With the notation $\widehat{f}$ for the $y z$-Fourier component of the base flow quantity $f$ of transverse wavenumber $k_{\perp}=\sqrt{k_{y}^{2}+k_{z}^{2}}$, this system amounts to

$$
\partial_{t} \widehat{\mathbf{U}}=\mathbf{L} \widehat{\mathbf{U}} \equiv-\mathbf{A} \partial_{m^{2}}^{2} \widehat{\mathbf{U}}-\mathbf{B} \partial_{m} \widehat{\mathbf{U}}-\mathbf{C} \widehat{\mathbf{U}},
$$

with $\widehat{\mathbf{U}}=\left[\widehat{\rho} \widehat{v} \widehat{d}_{\perp} \widehat{T}\right]^{\top}$, where $\widehat{d}_{\perp}$ denotes the Fourier component of the transverse divergence of the transverse velocity. The matrix $\mathbf{A}$ comprises a single non-zero coefficient, $A_{44}=C_{v}^{-1} \rho \Psi_{T^{\prime}}$, while the expressions of $\mathbf{B}$ and $\mathbf{C}$ are given by

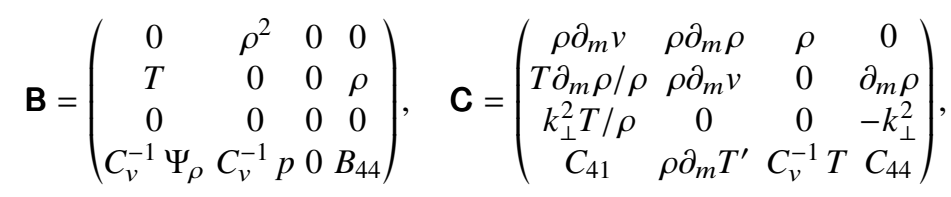

where

$$
\begin{gathered}
B_{44}=C_{v}^{-1}\left[\partial_{m}\left(\rho \Psi_{T^{\prime}}\right)+\Psi_{T}\right], \quad C_{41}=C_{v}^{-1}\left(\partial_{m} \Psi_{\rho}-\rho^{-1} \partial_{m} \varphi\right), \\
C_{44}=C_{v}^{-1}\left[\rho \partial_{m} v+\partial_{m} \Psi_{T}-k_{\perp}^{2} \rho^{-1} \Psi_{T^{\prime}}\right] .
\end{gathered}
$$

In these expressions $\Psi_{\rho}, \Psi_{T}$ and $\Psi_{T^{\prime}}$ stand for, respectively, the partial derivatives of the function $\Psi$ of (2) with respect to the density, temperature and temperature 
gradient. System (5) is of the incompletely parabolic type [15] since it comprises a parabolic subequation for the variable $\widehat{T}$ and a hyperbolic subsystem for the complementary variable vector $\left[\hat{\rho} \widehat{v} \widehat{d}_{\perp}\right]^{\top}$. Boundary conditions are provided by the

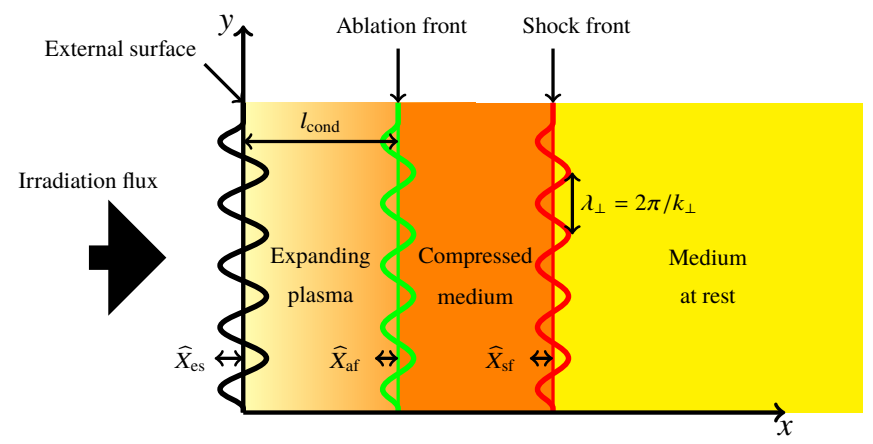

Fig. 2 Two-dimensional schematic view of a perturbed planar ablation wave. The length of the conduction region is denoted by $l_{\text {cond }}$ while $\widehat{X}_{a}, a=\mathrm{es,} \mathrm{af} \mathrm{or} \mathrm{sf,} \mathrm{denote} \mathrm{the} \mathrm{Fourier} \mathrm{components,}$ of transverse wavenumber $k_{\perp}=2 \pi / \lambda_{\perp}$, of the linear deformations of, respectively, the external surface, ablation front and shock front.

linearization (i), at the external surface of linear deformation $\widehat{X}_{\text {es }}$ (Fig. 2), of the continuity equations for the pressure and heat flux, and of the kinematic relation at a material surface, and (ii), at the shock front of linear deformation $\widehat{X}_{\mathrm{sf}}$, of the Rankine-Hugoniot jump relations [11]. From these boundary conditions, we infer, at any of the two boundaries $a=$ es or sf, the proper set of boundary conditions that defines a well-posed boundary value problem (BVP) for (5), namely: a scalar boundary condition for the parabolic subequation in $\widehat{T}$, say

$$
\widehat{\mathbf{B}}_{a}^{p}\left(\left.\partial_{m} \widehat{\mathbf{U}}\right|_{a},\left.\widehat{\mathbf{U}}\right|_{a}, \widehat{X}_{a}, \mathrm{~d}_{t} \widehat{X}_{a}\right)=0,
$$

as many boundary conditions as there are incoming waves of the hyperbolic subsystem at the boundary, or the system

$$
\widehat{\mathbf{B}}_{a}^{h}\left(\left.\widehat{\mathbf{U}}\right|_{a}, \widehat{X}_{a}, \mathrm{~d}_{t} \widehat{X}_{a}\right)=\mathbf{0}
$$

and an evolution equation for the boundary surface deformation $\widehat{X}_{a}$ written as

$$
f_{a}\left(\mathrm{~d}_{t} \widehat{X}_{a}, \widehat{X}_{a},\left.\widehat{\mathbf{U}}\right|_{a}\right)=0
$$

\section{Optimal perturbation}

The present non-modal stability analysis consists in finding the initial perturbation states $\left.\left(\widehat{\mathbf{U}}, \widehat{X}_{\mathrm{es}}, \widehat{X}_{\mathrm{sf}}\right)\right|_{t_{0}}$ that maximize an objective functional $\mathcal{J}$ depending only on 
the final states $\left.\left(\widehat{\mathbf{U}}, \widehat{X}_{\mathrm{es}}, \widehat{X}_{\mathrm{sf}}\right)\right|_{\mathcal{T}}$ where $\left(\widehat{\mathbf{U}}, \widehat{X}_{\mathrm{es}}, \widehat{X}_{\mathrm{sf}}\right)$ are solutions to (5)-(8) for zero external forcing and zero perturbations upstream to the shock front.

\subsection{Direct-adjoint method}

The optimization problem to be solved is presently formulated using the Lagrange multiplier technique [16]. In effect a Lagrange functional is defined from $\mathcal{J}$ augmented

(a)

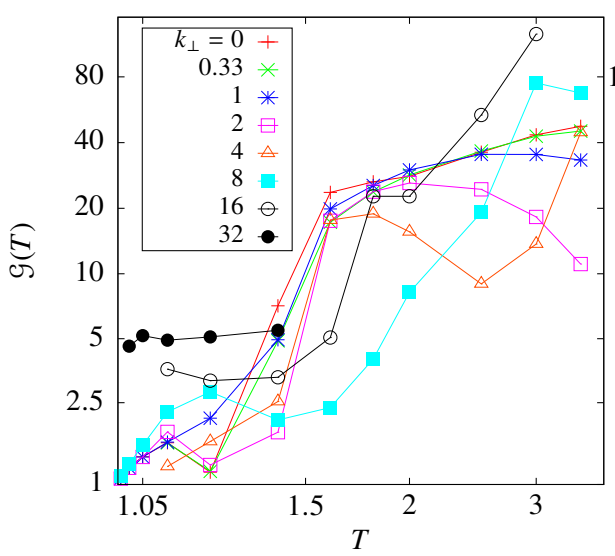

(b)

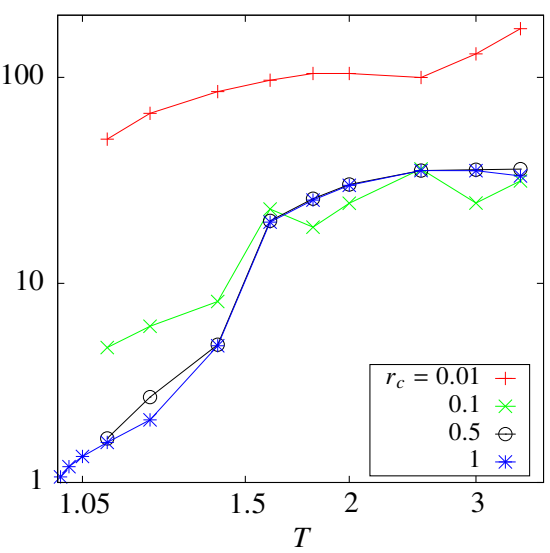

Fig. 3 Curves of the gain $\mathcal{G}\left(\mathcal{T}\right.$ ) for (a) $r_{c}=1$ and several values of $k_{\perp}$, (b) $k_{\perp}=1$ and several values of $r_{c}$.

by constraints enforcing the evolution equation (5), the sets of boundary conditions defining a well-posed BVP for (5), i.e. (6)-(8), and a normalization of the initial conditions, $\mathcal{J}\left(t_{0}\right)=K_{0}$, namely

$$
\begin{aligned}
\mathcal{L}=\mathcal{J}-\int_{0}^{m_{\mathrm{sf}}} & \int_{t_{0}}^{\mathcal{T}} \widehat{\mathbf{U}}^{\dagger \top}\left(\partial_{t} \widehat{\mathbf{U}}-\mathbf{L} \widehat{\mathbf{U}}\right) \mathrm{d} t \mathrm{~d} m \\
& -\sum_{a=\mathrm{es}, \mathrm{sf}} \int_{t_{0}}^{\mathcal{T}}\left(\widehat{\boldsymbol{v}}_{a}^{\dagger} \widehat{\mathbf{B}}_{a}^{h}+\widehat{\eta}_{a}^{\dagger} \widehat{\mathbf{B}}_{a}^{p}+\widehat{\mu}_{a}^{\dagger} f_{a}\right) \mathrm{d} t,-\beta^{\dagger}\left(\mathcal{J}\left(t_{0}\right)-K_{0}\right) .
\end{aligned}
$$

The optimum lies in the stationary points of $\mathcal{L}$. Differentiating with respect to the state variables yields the adjoint equation

$$
\begin{aligned}
\partial_{t} \widehat{\mathbf{U}}^{\dagger} & =\mathbf{L}^{\dagger} \widehat{\mathbf{U}}^{\dagger} \equiv \mathbf{A}^{\top} \partial_{m}^{2} \widehat{\mathbf{U}}^{\dagger}+\left(2 \partial_{m} \mathbf{A}-\mathbf{B}\right)^{\top} \partial_{m} \widehat{\mathbf{U}}^{\dagger}+\left(\partial_{m}^{2} \mathbf{A}-\partial_{m} \mathbf{B}+\mathbf{C}\right)^{\top} \widehat{\mathbf{U}}^{\dagger} \\
\widehat{\mathbf{B}}_{a}^{h^{\dagger}} & =\mathbf{0}, \quad \widehat{\mathbf{B}}_{a}^{p^{\dagger}}=0, \quad \mathrm{~d}_{t} \widehat{X}_{a}^{\dagger}=\dot{\hat{X}}_{a}^{\dagger},
\end{aligned}
$$


(a)

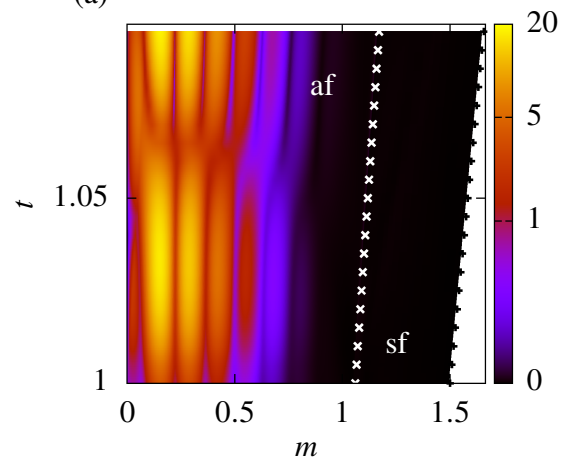

(b)

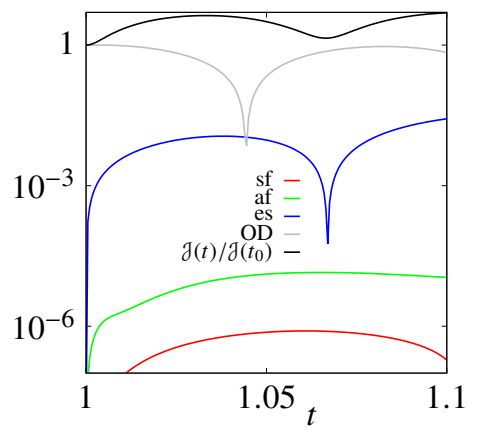

Fig. 4 Optimal gain for $\left(k_{\perp}, T\right)=(32,1.1), r_{c}=1$. (a) Chu's energy density $j$ in the variables $(m, t)$. (b) Deformations $\widehat{X}_{a}$ of the shock front (sf), ablation layer (af) and external surface (es), Chu's energy amplification $\left(\mathcal{J}(t) / \mathcal{J}\left(t_{0}\right)\right)$ and optical depth perturbation (OD) as functions of time.

where $\widehat{\mathbf{B}}_{a}^{h^{\dagger}}$ and $\widehat{\mathbf{B}}_{a}^{p^{\dagger}}$ represent the boundary condition operators for, respectively, the hyperbolic subsystem and parabolic subequation of (10a), $\dot{\hat{X}}_{a}^{\dagger}$ and $\widehat{X}_{a}^{\dagger}$ are scalar linear combinations of $\widehat{\boldsymbol{v}}_{a}^{\dagger}, \widehat{\eta}_{a}^{\dagger}$ and $\widehat{\mu}_{a}^{\dagger}$. The adjoint equation (10a) is well-posed for backward time integration from $t=\mathcal{T}$ to $t=t_{0}$ with terminal conditions

$$
\left.\widehat{\mathbf{U}}^{\dagger}\right|_{\mathcal{T}}=\nabla_{\left.\widehat{\mathbf{U}}\right|_{\mathcal{T}}} \mathcal{J},\left.\quad \widehat{X}_{a}^{\dagger}\right|_{\mathcal{T}}=\nabla_{\left.\widehat{X}_{a}\right|_{T}} \mathcal{J}
$$

Differentiation with respect to the control parameters, $\left.\widehat{\mathbf{U}}\right|_{t_{0}},\left.\widehat{X}_{a}\right|_{t_{0}}$, yields the optimality conditions

$$
\nabla_{\left.\widehat{\mathbf{U}}\right|_{t_{0}}} \mathcal{L}=\left.\widehat{\mathbf{U}}^{\dagger}\right|_{t_{0}}, \quad \nabla_{\left.\widehat{X}_{a}\right|_{t_{0}}} \mathcal{L}=\left.\widehat{X}_{a}^{\dagger}\right|_{t_{0}}
$$

where the right-hand-sides vanish at an optimum.

\section{Perturbation measure}

The functional $\mathcal{J}$ to be optimized is chosen to be defined after the energy of Chu [17], namely

$$
\mathcal{J}=\int_{0}^{m_{\mathrm{sf}}} \frac{j}{\rho} \mathrm{d} m, \quad \text { with } \quad j=\frac{1}{2}\left(\frac{T \widehat{\rho}^{2}}{\rho}+\rho \widehat{v}_{x}^{2}+\frac{\rho \widehat{d}_{\perp}^{2}}{k_{\perp}^{2}}+\frac{C_{v} \rho \widehat{T}^{2}}{T}\right),
$$

for $k_{\perp}>0$. This functional, being a semi-norm of the state variables, has to be supplemented by a complementary semi-norm which must be a norm for the kernel of $\mathcal{J}[18]$ and which we take to be

$$
\mathrm{J}^{*}=\left(X_{\mathrm{es}}^{2}+\widehat{X}_{\mathrm{sf}}^{2}\right) / 2
$$


so that $\mathcal{J}+\mathcal{J}^{*}$ is a full norm. Since our problem is linear, it is sufficient to restrict ourselves to the case $K_{0}=\mathcal{J}\left(t_{0}\right)+\mathcal{J}^{*}\left(t_{0}\right)=1$ in (9) and to vary the initial distribution of energy $r_{c}=\mathcal{J}\left(t_{0}\right) /\left(\mathcal{J}\left(t_{0}\right)+\mathcal{J}^{*}\left(t_{0}\right)\right)$ between 0 and 1 .

(a)

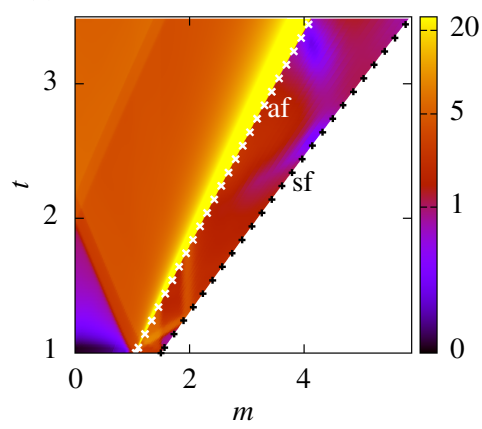

(b)

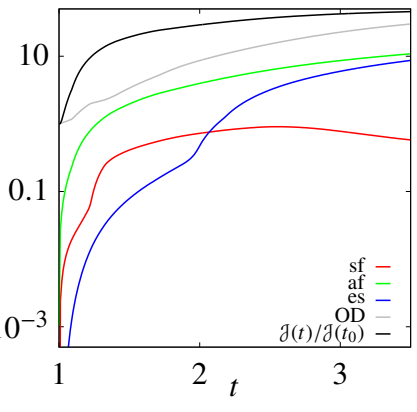

Fig. 5 Optimal gain for $\left(k_{\perp}, T\right)=(0.33,3.5), r_{c}=1$. (a) Chu's energy density $j$ in the variables $(m, t)$. (b) Deformations $\widehat{X}_{a}$ of the shock front (sf), ablation layer (af) and external surface (es), Chu's energy amplification $\left(\mathcal{\partial}(t) / \mathcal{\partial}\left(t_{0}\right)\right)$ and optical depth perturbation (OD) as functions of time.

\section{Optimization and numerical methods}

The direct and adjoint problems, (5) and (10), are solved iteratively using forward and backward temporal integrations, from a given starting initial condition. The terminal condition (11) is used to initialize the adjoint integration. Successive initial conditions of the direct problem are obtained from (12) by power iterations (e.g. see [19]). Numerical solutions to the direct system (5)-(8) and to the adjoint system (10), are obtained, in the space variable, using the same multidomain pseudospectral method as for the base flow and, in time, with a three-step implicit-explicit Runge-Kutta scheme. Boundary conditions are handled using a penalty method while matching conditions at subdomain interfaces are enforced exactly. The numerical codes for the direct and adjoint problems perform computations over each subdomain in parallel using the MPI paradigm with a single process per subdomain.

\subsection{Optimal initial perturbations}

The present analysis is performed for the self-similar ablative wave defined by the boundary parameters $\left(\mathcal{B}_{p}, \mathcal{B}_{\phi}\right)=(1.06,3.42)$ in (4). Optimization results have been computed with a spatial grid made of 189 subdomains with 50 collocation points each.

Optimal gains $\mathcal{G}(\mathcal{T})=\mathcal{J}(\mathcal{T}) / \mathcal{J}\left(t_{0}\right)$ larger than one have been obtained for all the transverse wavenumbers $k_{\perp}$, initial energy distributions $r_{c}$, and terminal times $\mathcal{T}$ that have been explored (Fig. 3). This fact denotes an inclination of the present ablation 
flow to significantly amplify perturbations. This amplification increases with $k_{\perp}$ for small terminal times, evidencing fast transient growth at small wavelengths (Fig. 3a). The initial distribution of perturbations in the deformations of the external surface and shock front, i.e. low values of $r_{c}$, leads to the strongest amplifications within the flow (Fig. 3b).

Two optimal growth mechanisms have been identified. For short final times $(1.01 \leqslant \mathcal{T} \leqslant 1.5)$, the OIP is concentrated within the tail of the conduction region and develops from local constructive interactions between acoustic and vorticity waves (Fig. 4a). The ablation layer and shock front are not involved in this mechanism as the terminal time $\mathcal{T}$ is shorter than the acoustic crossing time between these two locations (Fig. 4b). For larger terminal times, the OIP takes the form of forward acoustic and entropy signals in the post-shock region and perturbation energy grows from the interactions between the shock front and ablation layer resulting from the propagation of acoustic, entropy and vorticity waves across this region (Fig. 5). This mechanism is made possible since $\mathcal{T}$ exceeds several times the travel times of these waves across the post-shock region. At such terminal times, Chu's energy density is mostly located in the ablation layer (Fig. 5a). Temporal responses of optical depth perturbations, a quantity usually measured in experiments, appear to be uncorrelated to Chu's energy evolution (Figs. 4b, 5b) implying that experimental observation of perturbation amplification would require some other means of detection.

\section{Discussion and conclusion}

The first non-modal stability analysis of an ablation flow reported in this paper shows that ablation waves are susceptible to present transient perturbation growth for a wide range of perturbation characteristic lengths and time horizons. For the present self-similar ablation wave representative of the shock transit phase of an ICF pellet implosion, perturbation transient growths are found at all tested wavelengths and time horizons. Physical mechanisms responsible for such transient growths have been identified to be local constructive interactions between acoustic and vorticity waves within the flow conduction region at short time horizon, and interactions between the ablation layer and shock front for long terminal times. These findings contrast with previously known results established from normal mode analysis, simulations and dedicated experiments of selected "most dangerous" configurations, for which only large wavelengths are subject to a possible amplification whereas small wavelengths undergo damped oscillations [5, 20]. This confirms the interest and the necessity of non-modal stability analysis for ICF related flows. The present stability analyses has focused on the optimal perturbation problem. Optimal perturbations are found upon solving an optimization problem by means of a direct-adjoint method derived from the Lagrange multiplier technique. In the process, we identified the principle leading to a proper formulation of a Lagrange functional with boundary condition constrains for an incompletely parabolic system of equations with perturbed dynamical boundaries. This framework could easily be applied to receptivity 
problems and should stimulate the undertaking of non-modal analyses using ICF hydrocodes with "full physics".

\section{References}

1. S. Atzeni and J. Meyer-ter-Vehn. The physics of inertial fusion. Oxford University Press, Oxford, U.K., 2004.

2. Ya. B. Zel'dovich and Yu. P. Raizer. Physics of shock waves and high-temperature hydrodynamic phenomena. Academic Press, New-York, 1967.

3. J. Lindl et al. Review of the National Ignition Campaign 2009-2012. Phys. Plasmas, 21:020501, 2014.

4. V. Bychkov, M. Modestov, and C. K. Law. Combustion phenomena in modern physics: I. Inertial confinement fusion. Prog. Energy Combust. Sci., 47:32-59, 2015.

5. Y. Aglitskiy et al. Basic hyrdodynamics of Richtmyer-Meshkov-type growth and oscillations in the inertial confinement fusion-relevant conditions. Phil. Trans. R. Soc. A, 368:1739-1768, 2010.

6. K. S. Raman et al. An in-flight radiography platform to measure hydrodynamic instability growth in inertial confinement fusion capsules at the National Ignition Facility. Phys. Plasmas, 21:072710, 2014.

7. P. J. Schmid. Nonmodal stability theory. Annu. Rev. Fluid Mech., 39:129-162, 2007.

8. R. Marshak. Effect of radiation on shock wave behavior. Phys. Fluids, 1(1):24-29, 1958.

9. C. Boudesocque-Dubois, S. Gauthier, and J.-M. Clarisse. Self-similar solutions of unsteady ablation flows in inertial confinement fusion. J. Fluid Mech., 603:151-178, 2008.

10. J.-M. Clarisse et al. A hydrodynamic analysis of self-similar radiative ablation flows. J. Fluid Mech., 848:219-255, 2018.

11. J.-M. Clarisse, C. Boudesocque-Dubois, and S. Gauthier. Linear perturbation response of self-similar ablative flows relevant to inertial confinement fusion. J. Fluid Mech., 609:1-48, 2008.

12. J.-M. Clarisse et al. Transient effects in unstable ablation fronts and mixing layers in HEDP. Phys. Scr., 91:074005, 2016.

13. F. Abéguilé, C. Boudesocque-Dubois, J.-M. Clarisse, S. Gauthier, and Y. Saillard. Linear perturbation amplification in self-similar ablation flows of inertial confinement fusion. Phys. Rev. Lett., 97:035002, 2006.

14. C. Boudesocque-Dubois, V. Lombard, S. Gauthier, and J.-M. Clarisse. An adaptive multidomain Chebyshev method for nonlinear eigenvalue problems: Application to self-similar solutions of gas dynamics equations with nonlinear heat conduction. J. Comput. Phys., 235:723-741, 2013.

15. J. C. Strikwerda. Initial boundary value problems for incompletely parabolic systems. Commun. Pure Appl. Math., XXX:797-822, 1977.

16. M. D. Gunzburger. Introduction into mathematical aspect of flow control and optimization. In von Karman Institute for Fluid Dynamics, editor, Lecture Series 1997-05 on Inverse Design and Optimisation Methods. April 1997.

17. B.-T. Chu. On the energy transfer to small disturbances in fluid flow (parti i). Acta Mechanica, 1:215-234, 1965.

18. D. P. G Foures, C. P. Caulfield, and P. J. Schmid. Variational framework for flow optimization using seminorm constraints. Phys. Rev. E, 86(2):026306, Aug 2012.

19. P. Corbett and A. Bottaro. Optimal perturbations for boundary layers subject to stream-wise pressure gradient. Phys. Fluids, 12(1):120-130, 2000.

20. V. N. Goncharov et al. A model of laser imprinting. Phys. Plasmas, 7(5):2062-2068, 2000. 